\title{
On the Behavior of the Infinite Restricted Boltzmann Machine for Clustering
}

\author{
Nikolas A. Huhnstock \\ University of Skövde \\ Skövde, Sweden \\ Maria Riveiro \\ University of Skövde \\ Skövde, Sweden
}

\author{
Alexander Karlsson \\ University of Skövde \\ Skövde, Sweden \\ H. Joe Steinhauer \\ University of Skövde \\ Skövde, Sweden
}

\begin{abstract}
Clustering is a core problem within a wide range of research disciplines ranging from machine learning and data mining to classical statistics. A group of clustering approaches so-called nonparametric methods, aims to cluster a set of entities into a beforehand unspecified and unknown number of clusters, making potentially expensive pre-analysis of data obsolete. In this paper, the recently, by Côté and Larochelle introduced infinite Restricted Boltzmann Machine that has the ability to self-regulate its number of hidden parameters is adapted to the problem of clustering by the introduction of two basic cluster membership assumptions. A descriptive study of the influence of several regularization and sparsity settings on the clustering behavior is presented and results are discussed. The results show that sparsity is a key adaption when using the iRBM for clustering that improves both the clustering performances as well as the number of identified clusters.
\end{abstract}

\section{CCS CONCEPTS}

- Computing methodologies $\rightarrow$ Unsupervised learning; Cluster analysis;

\section{KEYWORDS}

clustering, unsupervised, machine learning, restricted boltzmann machine

\section{ACM Reference Format:}

Nikolas A. Huhnstock, Alexander Karlsson, Maria Riveiro, and H. Joe Steinhauer. 2018. On the Behavior of the Infinite Restricted Boltzmann Machine for Clustering. In SAC 2018: SAC 2018: Symposium on Applied Computing , April 9-13, 2018, Pau, France. ACM, New York, NY, USA, 10 pages. https://doi.org/10.1145/3167132.3167183

\section{INTRODUCTION}

Unsupervised Learning [10] describes the problem of finding meaningful patterns in a set of unlabeled data. Clustering is one specialization of unsupervised learning trying to discover groups (clusters)

Permission to make digital or hard copies of part or all of this work for personal or classroom use is granted without fee provided that copies are not made or distributed for profit or commercial advantage and that copies bear this notice and the full citation on the first page. Copyrights for third-party components of this work must be honored. For all other uses, contact the owner/author(s).

SAC 2018, April 9-13, 2018, Pau, France

(C) 2018 Copyright held by the owner/author(s).

ACM ISBN 978-1-4503-5191-1/18/04.

https://doi.org/10.1145/3167132.3167183 of similar patterns within the data [2]. Typically clusters are considered to be meaningful if the entities they enclose are more related ${ }^{1}$ to items within the same cluster than to entities of other clusters [10].

Widely used approaches for clustering are among others, kmeans and k-medoids [10], which form clusters based on predefined distance functions, e.g., Euclidean, in the objects feature space. An increase in distance is interpreted as a decrease in similarity [9]. Statistical methods are often based on the assumption that entities of a cluster are sample populations from an underlying distribution. Mixture Models (MM) [2] describe, for example, each cluster as a mixture of, e.g., Gaussian distributions assigning entities to the mixture's component with highest posterior probability[2].

The major drawback of both types of methods is that they take the number of clusters entities should be assigned to as an input. This parameter has either be decided by an expert user or to be determined during pre-analysis of the data. Thus, the performance of these approaches to clustering is dependent on an external method, which often involves the evaluation of a metric for a range of target number of clusters, such as the silhouette method [24] or the gap statistic [33].

Today, data is collected from more and more sources at a remarkable pace (Big data, Internet of Things, Industry 4.0). Such data might lead to scenarios where no experts with comprehensible knowledge about the data are available and where therefore pre-analysis comes at enormous additional costs. In addition, in a scenario where data not received in batches but in streams, predetermination of the number of clusters is made infeasible and static methods become inapplicable.

Recently, it has been shown that the Restricted Boltzmann Machine (RBM) [29] is a viable model that has, among others been applied to a range of problems in multiple domains, e.g., dimensionality reduction[13], image classification [30], recommender systems [25] and in the context of clustering.

In clustering applications, the RBM has been used as a feature extraction or dimensionality reduction tool [7] either by itself or in the form of a Deep Belief Network (DBN), where multiple RBMs are stacked on top of each other [26, 35]. However, in all these approaches the RBM relied on additional methods, e.g., MMs or Kmeans, to perform the actual task of clustering or was used to solve only supervised / semi-supervised clustering, i.e., classification. In spite of the fact that the technique of stacking models has shown to improve classification results significantly [18], it increases as well

\footnotetext{
${ }^{1}$ Multiple metrics exist to asses similarity of two entries, e.g., cosine-similarity.
} 
the number of parameters of the overall model. A problem with this approach is that occurring interdependencies between the chosen methods have to be considered when composing the model in order to achieve an increase in performance [15]. The combination of multiple methods comes with the risk of increasing the complexity of the approach, which is in general not desirable following Occam's razor [2]. As a self-contained clustering method, the RBM has been applied by Steinhauer et al. [31] to the problem of finding rootcause relations in a small abstract model of a telecommunication network.

In this work we explore the possibility of using an adapted version of the RBM, the infinite Restricted Boltzmann Machine (iRBM), that has been introduced by Côté and Larochelle [3], to address the problem of unsupervised clustering. Thanks to an ordered weight training procedure and an adapted energy function the iRBM is capable of self-adjusting its hidden layer size to the data at hand. This adaptive behavior is important in an unsupervised clustering setting where we can not rely on labeled information during training, as in $[6,16]$. Furthermore, we aim for a comprehensive and self-sufficient approach, which does not rely on additional methods or experts to determine the number of clusters. The idea is similar to Bayesian non-parametrics [32] where the Dirichlet process is the basic building block for several clustering methods, e.g., Dirichlet Process Mixture Models [1].

Two assumptions are introduced in order to (1) interpret the iRBMs hidden layer activations as cluster assignments and (2) enable the model to adapt the number of available clusters directly to the data. Based on that, we conduct several experiments to study the effect of different regularization and sparsity settings of the iRBMs clustering behavior with the use of descriptive statistics.

This paper is structured as follows: Section 2 gives the theoretical background of the iRBM followed by a review of the related work in Section 3. Section 4 introduces how the iRBM is adapted to the problem of clustering. The conducted experiments are described in Section 5 followed by a discussion of the obtained results in Section 6. Finally, Section 7 concludes the paper.

\section{PRELIMINARIES}

\section{Infinite Restricted Boltzmann Machine (iRBM)}

The iRBM [3] differs from the basic RBM in two aspects: firstly it builds upon the ordered hidden unit training from the ordered RBM (oRBM) [3] and secondly, it extends the hidden layer size of the oRBM to an, in theory, infinite number of units [3]. This section describes the theoretical foundations from which the iRBM is derived.

A RBM is a Markov Random Field (MRF) [4] associated with an undirected bipartite graph $\mathcal{G}=(\mathcal{V}, \mathcal{H})$, see Figure 1 . In the same way, as a MRF describes a set of random variables, the RBMs visible units $v$ and hidden units $h$ are only conditionally dependent on neighboring units. The special bipartite structure separating the two kinds of units into layers allows each unit to be only dependent on the other layer's units, as expressed in Equations (3) and (4). Considering the binary RBM, where units in the visible layer take values $v \in\{0,1\}^{n}$ and units in the hidden layer $h \in\{0,1\}^{m}$, the

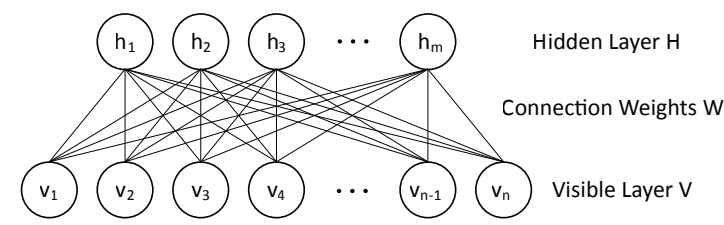

Figure 1: Graph representation of a Restricted Boltzmann machine illustrating the two bipartite layers consisting of $n$ and $m$ fixed number of units per layer. The bottom layer consists of visible units and the top layer of hidden units.

joint probability distribution is given by

$$
P(\mathbf{v}, \mathbf{h})=\frac{1}{Z(\mathbf{v}, \mathbf{h})} e^{-E(\mathbf{v}, \mathbf{h})}
$$

with a so called energy function

$$
E(\mathbf{v}, \mathbf{h})=-\sum_{i=1}^{n} \sum_{j=1}^{m} w_{i j} v_{i} h_{j}-\sum_{i=1}^{n} b_{i} v_{i}-\sum_{j=1}^{m} c_{j} h_{j} .
$$

The term $Z(\mathbf{v}, \mathbf{h})$ is called the partition function and is defined as $Z(\mathbf{v}, \mathbf{h})=\sum_{v \in \mathcal{V}, h \in \mathcal{H}} e^{-E(\mathbf{v}, \mathbf{h})}$. The conditional probabilities are given by

$$
P(\mathbf{h} \mid \mathbf{v})=\prod_{j=1}^{m} P\left(h_{j}=1 \mid \mathbf{v}\right)
$$

and

$$
P(\mathbf{v} \mid \mathbf{h})=\prod_{i=1}^{n} P\left(v_{i}=1 \mid \mathbf{h}\right) .
$$

The probability of a single unit being active (state equal 1) is defined as

$$
P\left(h_{j}=1 \mid \mathbf{v}\right)=\sigma\left(\sum_{i=1}^{n} v_{i} w_{i j}+c_{j}\right)
$$

and

$$
P\left(v_{i}=1 \mid \mathbf{h}\right)=\sigma\left(\sum_{j=1}^{m} h_{j} w_{i j}+b_{i}\right)
$$

for visible and hidden units respectively, with $\sigma(x)=\frac{1}{1+e^{-x}}$.

The first step in the transformation into the iRBM is to introduce the idea that the model has a finite but theoretically unlimited number of hidden units while the number of visible units $n$ is still defined by the input data $\mathcal{D}^{n \times d}$. The number of actually considered hidden units is given by $z \in \mathbb{N}$, which is conditionally dependent on the model's visible state $(v)$ and thus defines the number of hidden units contributing to the model's energy. For each selected hidden unit $h_{j}$ there is an energy penalty $\beta_{j}$ added to the energy function which counteracts the infinite accumulation of units. The extended energy function takes the following form

$$
\begin{aligned}
E(\mathbf{v}, \mathbf{h}, z)=-\sum_{i=1}^{n} \sum_{j=1}^{z} w_{i j} v_{i} h_{j}-\sum_{i=1}^{n} b_{i} v_{i} & \\
& -\sum_{j=1}^{z}\left(c_{j} h_{j}-\beta_{j}\right) .
\end{aligned}
$$


The penalty term $\beta_{j}$ is parametrized as $\beta_{j}=\beta$ soft $_{+}\left(c_{j}\right)$ with $\beta$ being the common penalty hyper-parameter over all units and soft $_{+}(x)=\ln \left(1+e^{x}\right)$. Having $\beta_{j}$ defined on each unit's bias implicitly forces the model to learn better weights $w_{i j}$ in order to increase its size [3].

Since the model's joint probability distribution is defined regarding its energy, Equations (1), (3), (4), (5) and (6) have to be adapted as well.

The joint probability over $\mathbf{v}, \mathbf{h}$ and $z$ becomes

$$
P(\mathbf{v}, \mathbf{h}, z)=\frac{1}{Z(\mathbf{v}, \mathbf{h}, z)} e^{-E(\mathbf{v}, \mathbf{h}, z)}
$$

with $Z(\mathbf{v}, \mathbf{h}, z)=\sum_{\mathbf{v} \in \mathcal{V}, \mathbf{h} \in \mathcal{H}_{z}} e^{-E(\mathbf{v}, \mathbf{h}, z)}$ where $\mathcal{H}_{z}=\{\mathbf{h} \in \mathcal{H}$ $\left.h_{j}=0 \forall j>z\right\}$ defines the set of legal values of $\mathbf{h}$. This formalizes the implicit assumption that hyper-parameters associated with unselected hidden units, i.e., $\left\{h_{j}, \forall j>z\right\}$, are zero and that these units thus do not contribute to the model's energy at the specific configuration $(\mathbf{v}, \mathbf{h}, z)$.

The value of $z$ is context dependent and thus varies dependent on the input to the model. The conditional probability of $z$ is defined with use of the model's free energy, which itself is derived by marginalizing the numerator of Equation (8) over its hidden units leading to

$$
F(\mathbf{v}, z)=-\sum_{i=1}^{n} b_{i} v_{i}-\sum_{t=1}^{z}\left(\operatorname{soft}_{+}\left(\sum_{i=1}^{n} w_{i t} v_{i}+c_{t}\right)-\beta_{t}\right) .
$$

With the free energy defined, the conditional probability of $z$, given a state $\mathbf{v}$ of the visible layer, is

$$
P(z \mid \mathbf{v})=\frac{e^{-F(\mathbf{v}, z)}}{Z(\mathbf{v})} .
$$

The normalization function $Z(\mathbf{v})$ is given by $Z(\mathbf{v})=\sum_{z=1}^{\infty} e^{-F(\mathbf{v}, \mathbf{z})}$. This term becomes tractable considering that for a given set of inputs $\mathcal{D}=\left\{x_{1}, \ldots, x_{d}\right\}$ there will be $z^{*}=$ $\operatorname{argmax}_{z \in \mathbb{N}}(P(z \mid \mathbf{v})), \forall \mathbf{v} \in \mathcal{D}$ sampled using Equation (10), where units with indices greater than $z^{*}$ will, per definition, have zero weights and bias. The infinite sum of $Z(\mathrm{v})$ can be split at $z^{*}$ into two sums: the first sum $\sum_{z=1}^{z^{*}} e^{-F(\mathbf{v}, \mathbf{z})}$ can be analytically computed. The second sum, $\sum_{z=1}^{z^{*}+1} e^{-F(\mathbf{v}, \mathbf{z})}$ degenerates to a geometric series leading to a finite partition function, given it is ensured that $\beta$ is greater than one, as shown by Côté and Larochelle [3].

The conditional probabilities of each unit being active, Equations (3) and (4), have to incorporate the changing number of hidden units as well:

$$
\begin{aligned}
& P\left(h_{j}=1 \mid \mathbf{v}, z\right)= \begin{cases}\sigma\left(\sum_{i=1}^{n} v_{i} w_{i j}+c_{j}\right), & j \leq z \\
0, & \text { otherwise }\end{cases} \\
& P\left(v_{i}=1 \mid \mathbf{h}, z\right)=\sigma\left(\sum_{j=1}^{z} h_{j} w_{i j}+b_{i}\right)
\end{aligned}
$$

\section{Training of an iRBM}

The iRBM is trained by lowering the model's energy for a dataset $\mathcal{D} \in\{0,1\}^{n \times d}$, with d samples, and raising the probability of the data given the model. This is achieved by adapting the model's weights and biases using contrastive divergence (CD) [11] to estimate the gradients of the model's free energy:

$$
F(\mathbf{v})=-\ln \sum_{z=1}^{z^{*}} e^{-F(\mathbf{v}, z)}
$$

where $z^{*}=\operatorname{argmax}_{z \in \mathbb{N}}(P(z \mid \mathbf{v})), \forall \mathbf{v} \in \mathcal{D}$ and $F(\mathbf{v}, z)$ as in Equation (9). The partial derivatives w.r.t. the model's parameters are given by

$$
\begin{aligned}
& \frac{\partial F(\mathbf{v}, z)}{\partial w_{i j}}= \begin{cases}\sigma\left(\sum_{i=1}^{n} w_{i j} v_{i}+c_{t}\right) v_{i}, & j \leq z \\
0, & \text { otherwise }\end{cases} \\
& \frac{\partial F(\mathbf{v}, z)}{\partial c_{j}}= \begin{cases}\sigma\left(\sum_{i=1}^{n} w_{i t} v_{i}+c_{t}\right)-\beta \sigma\left(c_{j}\right), & j \leq z \\
0, & \text { otherwise }\end{cases} \\
& \frac{\partial F(\mathbf{v}, z)}{\partial b_{i}}=-v_{i}
\end{aligned}
$$

\section{Regularization and Sparsity}

Regularization, in general, describes methods that aim to control over-fitting. Two commonly deployed methods are $L_{1}$ and $L_{2}$ regularization also referred to as weight decay, where a weight dependent term is used to penalize weights. The general expression for regularization penalties is defined as [2]:

$$
L_{q}\left(w_{i j}\right)=\frac{\lambda}{q} \sum_{i=1}^{n} \sum_{j=1}^{m}\left|w_{i j}\right|^{q}
$$

where $\lambda$ is the weight cost parameter, used to fine-tune the regularizing effect. The only difference between the two types of regularization, which are added to the parameter updates during training, is the choice of parameter $q$. Setting $q=1$ realizes $L_{1}$ weight decay, having the property of forcing the majority of parameters towards zero, while a few will grow larger and thus, introducing sparseness into the model. Similarly, $L_{2}$ weight decay is obtained by setting $q=2$. This setting penalizes larger weights more than smaller ones, forcing the weights to be more similar to each other and has, therefore a smoothening influence on the weights.

A third type of regularization, which is deployed in the experiments presented in this paper, is a linear combination of the former two, referred to as $L_{12}$ regularization and also known as elastic net regularization [39]. $L_{12}$ regularization aims to combine the sparsity inducing properties of $L_{1}$ regularization and the grouping influence of $L_{2}$ regularization.

A different type of regularization, introduced by Nair and Hinton [21], which is not directly based on the weights, aims to encourage sparse hidden activations by reducing ${ }^{2}$ the mean probability of a unit being active. Given the actual activation distribution $q$ and a desired target activation distribution $p$, they proposed to use the cross-entropy of the two given by $p \log q+(1-p) \log (1-q)$ as penalty. Forcing sparse representations is a common technique in unsupervised learning methods [23] since it introduces robustness against noise and is "especially advantageous for classifiers because classification is more likely to be easier in higher dimensional spaces" [23].

\footnotetext{
${ }^{2}$ It might as well raise unit's mean activation when the current unit's mean activation $q$ happens to be lower than the chosen target activation $p$, leading to a unit reviving property [21].
} 


\section{RELATED WORK}

In this section, related research that deployed the RBM for clustering or classification, as well as related approaches for unsupervised clustering, are reviewed.

The classification RBM (classRBM), presented in [12,17], includes an additional label layer and has proven to be a competitive model to solve supervised clustering problems, which showcases the RBM's potential to be used as a clustering approach. This model however only solves supervised and semi-supervised problems and thus relies on labeled data during training.

A combination of multiple RBMs, each trained on a single class was proposed by Schmah et al. [28]. Here cluster membership is determined by the model that most likely could have generated the test sample. The iRBM can as well be interpreted as a composition of multiple RBMs each having a different number of hidden units. Which model to be used in this manner is determined by Equation (8). The main difference is that Schmah et al. [28] train a separate model for each cluster which means: Firstly, they have to know the number of clusters in advance. Secondly, that they have to train a complete set of parameters for each model. Because the iRBM shares the weights between all models, this results in training all models at the same time. And thirdly, the model developed by Schmah et al. [28] is also reliant on labeled training data.

Salama et al. [26] used a 3-layer DBN with a single output unit to cluster data. Each cluster found had to be manually associated with an interval of the single unit's output. Although their method is able to differentiate between three clusters, the overlapping outputs considering a dataset with four clusters is already a problem which will most likely increase for data sets where a higher number of clusters is appropriate to be found. Additionally, the number of layers and number of units per layer had to be defined manually in advance. In comparison, the iRBM identifies the number of hidden units by self-adjusting its structure depending on the data.

The Dirichlet Process (DP) is the baseline approach to nonparametric clustering, and consists of an infinite dimensional generalization of a Bayesian mixture model with Dirichlet prior [32]. In a so called Dirichlet Process Mixture Model (DPMM) components are drawn from a base distribution $\mathrm{H}$, where each component is assumed to describe a subset of the data. The number of drawn components, which can be interpreted as clusters is solely logarithmic dependent on the number of data and the hyper-parameter $\alpha$. The DPMM has similar self-adapting properties as the iRBM but is based on strong assumptions such as the choice of base distribution.

The affinity propagation [5] algorithm finds the number of clusters by identifying prototypes from the data. The prototypes are determined from an iterative message passing procedure with the use of pairwise similarities of data points. However, distance based similarities do not perform well on high dimensional data as they suffer from the curse of dimensionality $[8,38]$.

\section{APPROACHING CLUSTERING USING THE IRBM}

To use the iRBM as a clustering method, we make the following assumptions:

i Related entities $\left(\mathbf{x}_{a}, \mathbf{x}_{b}\right) \in \mathcal{D}^{n \times d}$ will be associated with a similar hidden state, i.e., $P\left(\mathbf{h}^{a} \mid \mathbf{x}_{a}\right) \simeq P\left(\mathbf{h}^{b} \mid \mathbf{x}_{b}\right)$. ii The dominant hidden unit $h_{j^{*}}=\max _{1 \leq j \leq m} P\left(h_{j}=1 \mid \mathbf{x}\right), \mathbf{x} \in \mathcal{D}$ will determine the cluster-membership of sample $\mathbf{x}$ sufficiently.

Assumption (i) bases on the fact that an RBM's hidden units capture dependencies between observed visible units [4]. Considering that entities of one cluster are supposed to be more similar to each other than to entities of other clusters $[9,36]$, these entities are likely to exhibit a common pattern which will be reflected in the data $\mathcal{D}$. During training the model's weights are adjusted, so that the model becomes more likely to have generated the data. The hidden units associated with learned patterns are therefore more likely to be active, according to Equation (11), when a test sample $\mathbf{x}_{t}$ complies to their encoded pattern. The weights of a trained model can be interpreted as a filter between visible and hidden layer. Thus a subset $C_{p} \in \mathcal{D}$ of samples $\left(\mathbf{x}_{1}, \ldots, \mathbf{x}_{u}\right)$ embodying a common pattern will comply to similar filters and therefore tend to generate similar hidden states.

Assumption (ii) manifests the direct connection between the model's clustering capabilities and its hidden layer size. It bounds the maximum number of clusters that the model is able to differentiate to the model's hidden layer size: considering a model having $m$ hidden units it is able to partition $\mathcal{D}$ into a maximal number of $m$ clusters. While imposing an upper bound on the number of clusters, it also functions as a method to adapt the number of clusters to the data, making expert knowledge or previous analyzations obsolete.

Through the learned (weight based) filters hidden units specialize on patterns present within $\mathcal{D}$. One such filter, defined by weights w. , associated with a specialized hidden unit $h_{p}$, acts as barrier activating $h_{p}$ proportional to the match between $\mathbf{w} \cdot p$ (learned filter) and test sample $\mathbf{x}$. We denote the most active hidden unit for a test sample $\mathbf{x}$ as $h_{p^{*}}=\operatorname{argmax} h_{p} \forall h_{p} \in \mathcal{H}$. This unit $h_{p^{*}}$ can only be the most active hidden unit when its associated filter $\mathbf{w} \cdot p^{*}$ is the one most compliant with $\mathbf{x}$. Thus $h_{p^{*}}$ encodes the most discriminative pattern for $\mathbf{x}$ and will in combination with Assumption (i) enclose samples embodying patterns equal to $\mathbf{x}$ and therefore form clusters. In other words, all inputs that activate the same dominant unit are assigned to the same cluster.

Additionally, Assumption (ii) implies that we are aiming for a hard partitioning of $\mathcal{D}$, where each entity is assigned to exactly one cluster [36].

Using the iRBM to solve the problem of unsupervised clustering we first note that the model does not need to learn an optimal representation. It is sufficient to capture the rough underlying patterns as long as the model is not used to recreate the data with minimal error, as when used as a generative model [4].

\section{EMPIRICAL EVALUATION}

To assess the iRBM's clustering capabilities, trained models are evaluated on a test set using two measures that are typically used to assess clustering performance: Adjusted Mutual Information (AMI) [34] and Adjusted Rand Index (ARI) [14]. The former is a commonly used measure, [20, 22, 35, 37], expressing dependencies of the resulting clusters and the true labels. While the latter is chosen because it is especially suited to multi-class clustering problems [27]. 


\subsection{Experiment Design}

The AMI measures the amount of statistical information and expresses the (for chance adjusted) mutual dependence between two random variables or, in the case of clustering, "the amount of statistical information shared by the random variables representing the cluster assignments $[C]$ and the user-labeled class assignments $[K]$ of the data points" [22]. It is defined as [34]:

$$
\operatorname{AMI}(C, K)=\frac{I(C ; K)-\mathrm{E}[I(C, K)]}{\max \{H(C), H(K)\}-\mathrm{E}[I(C, K)]},
$$

where $I(X ; Y)=H(X)-H(X \mid Y)$ defines the common mutual information of random variables $X$ and $Y, H(X)$ is the Shannon entropy given by $H(X)=\mathbf{E}[-\ln (P(X))]$.

The ARI is based on counting the number of entities that are found in the same cluster and have the same (known) label, defined by [14] as:

$$
\operatorname{ARI}(C, K)=\frac{\sum_{i j}\left(\begin{array}{c}
n_{i j} \\
2
\end{array}\right)-\frac{\sum_{i}\left(\begin{array}{c}
n_{i} \cdot \\
2
\end{array}\right) \sum_{j}\left(\begin{array}{c}
n_{\cdot j} \\
2
\end{array}\right)}{\frac{\left(\begin{array}{c}
n \\
2
\end{array}\right)}{\sum_{i}\left(\begin{array}{c}
n_{i} \cdot \\
2
\end{array}\right)+\sum_{j}\left(\begin{array}{c}
n_{\cdot j} \\
2
\end{array}\right)}}-\frac{\sum_{i}\left(\begin{array}{c}
n_{i} \\
2
\end{array}\right) \sum_{j}\left(\begin{array}{c}
n_{\cdot j} \\
2
\end{array}\right)}{2},}{\left(\begin{array}{c}
n \\
2
\end{array}\right)},
$$

where $n_{i j}$ is the number of entities of the $j$-th cluster of $C$ sharing the $i$-th label of $K$, while $n_{i}$. and $n . j$ define the number of entities assigned to label $i$ and cluster $j$ respectively.

To evaluate the behavior and performance of the iRBM we conducted experiments using the MNIST dataset [19], which offers a high-dimensional real-world clustering problem and thus a suitable candidate to give insight into the iRBM's clustering capabilities.

Since the goal of clustering, in general, is to give meaningful insight into the data [36] the number of resulting clusters found is an important factor when interpreting the results. Therefore the number of identified clusters is part of our behavioral study.

The MNIST dataset, introduced in [19], offers a large corpus of labeled high-dimensional real world data consisting of handwritten digits ranging from $0-9$ in the form of 28 by 28 grayscale images. For our experiments, we used a binary representation of the dataset, where each original grayscale pixel $\in[0,256]$ is set to either 0 or 1 when its value is greater or lower than 128 respectively.

In the following, four experiments are described to analyze the clustering behavior of the iRBM are described.

Experiment 1. The aim of the first experiment is to study if Assumption (ii) does work as hypothesized in Section 4. Therefore, we compare two binary RBMs both with fixed hidden layer size of 200 units but with different cluster mappings. The first model uses the dominant unit approach, as described in Section 4, whereas the second model uses all hidden units to determine cluster membership - inputs $\left(\mathbf{x}^{a}, \mathbf{x}^{b}\right)$ producing the same hidden states $\left(\mathbf{h}^{a}=\mathbf{h}^{b}\right)$ are assigned to the same cluster. Hidden states are determined by (3).

Experiment 2. In the second experiment, six standard RBMs with fixed hidden layer size of 5, 10, 25, 50, 100 and 200 are trained on the MNIST data set. No regularization or sparsity is deployed. The aim of this experiment is to show the relation between the size of the hidden layers and the clustering performance, e.g., AMI and
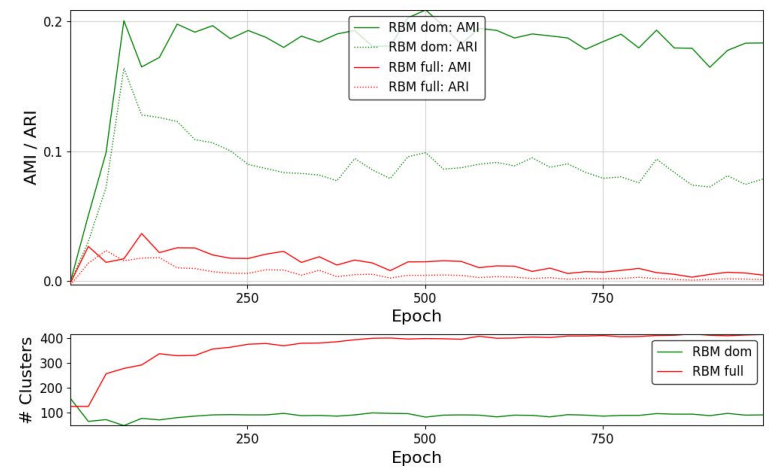

Figure 2: Results of the first experiment. (top) The plot shows the AMI and ARI scores of two RBMs. The first model (RBM dom) uses the dominant unit approach, whereas the second model (RBM full) uses all hidden units to determine cluster membership. Scores are evaluated on a test set over the course of a training procedure (1000 Epochs). Models are trained on the binarized MNIST dataset. (bottom) The number of identified clusters for each model.

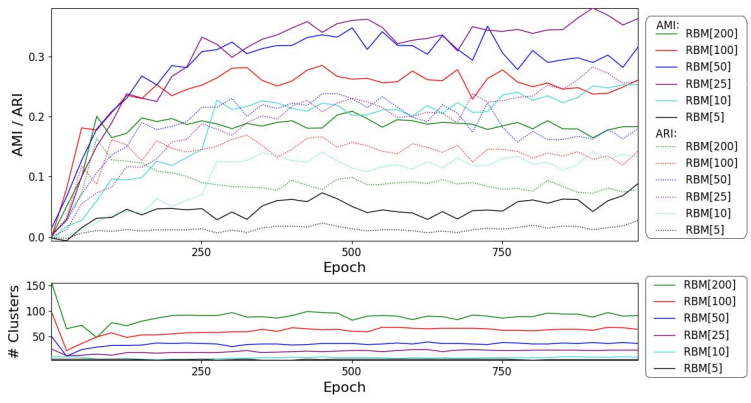

Figure 3: Results of second the experiment. (top) The plot shows the AMI and ARI scores of 6 RBM, having 5, 10, 25, 50, 100 and 200 hidden units respectively. Scores are evaluated on a test set over the course of a training procedure (1000 Epochs) trained on the binarized MNIST dataset. (bottom) The number of identified clusters for each model.

ARI score as well as the number of identified clusters during the process of learning.

The experiment's outcome should show the influence of the hidden layer size on the clustering performance and highlight that the number of hidden units is a sensible parameter to set.

Experiment 3. The objective of the third experiment is to examine how different regularization and sparsity settings affect the clustering. Therefore one iRBM has been trained and evaluated for each possible combination of sparsity and regularization, while $\beta$ is kept constant for all of them. The sparsity target is set to $p=0.1$ as suggested by Ranzato et al. [23].

Experiment 4. In the fourth experiment, the influence of energy penalty $\beta$ on the resulting clusters is studied. The described measures are evaluated for multiple $\beta \in] 1,2]$. We report mean and 
standard deviation (std) of ten training and evaluation iterations, as well as the average number of identified clusters.

\subsection{Results}

Experiment 1. The results of the first experiment, illustrated in Figure 2, show that as expected, Assumption (ii) successfully reduces the number of produced clusters while reaching higher AMI scores ARI scores. Not surprisingly does the model using the complete hidden layer to determine cluster membership lead to a high number of generated clusters (400 clusters) compared to the model deploying the dominant unit approach described in Section 4 (75 clusters). The ARI scores indicate that the models start to overfit regarding clustering, whereas the AMI does not indicate such behavior. Considering the usefulness of the produced clusterings taking into account that we know the data set consist of ten different labels - as argued in Section 5.1 it's desirable to have fewer but more expressive clusters than a vast number of atomic clusters.

Experiment 2. The results of the second experiment, depicted in Figure 3, show that the models are learning better clusters as training progresses, depicted by rising AMI and ARI values (Figure 3 , top, dashed and solid lines respectively). At the beginning of the training all models identify a huge number of clusters but as training progresses the number of identified clusters shrinks, (Figure 3, bottom). This corresponds to the fact that the models' initial random filters are not yet adapted to determine clusters generating too many resulting clusters. The six trained models showcase the influence of the number of hidden units on the resulting clustering. RBMs with five and ten hidden units reach low scores compared to models with more hidden units. Scores of models having more than 50 units are penalized by the high number of identified clusters. In summary, experiment 2 depicts the role of the hidden layer's size regarding clustering performance. Especially, the number of generated clusters is sensible to the size of the hidden layer - which can't be set without pre-analysis of the data. From this experiment the recommended number of hidden units would be 25 to 50 . The results of the following two experiments will show if the iRBM is capable to self-adjust it's hidden layer size to the data and generate useful clusters.

Experiment 3. The results of the third experiment are divided into two parts in order to describe the behavior in greater detail and (mostly) isolated from interfering influences. The first part, deals with the influence of sparsity, while the second part focusses on the influence of regularization. The parts are summarized in Figure 4 and 5 respectively.

The influence of sparsity on the iRBM's clustering behavior is depicted in Figure 4, consisting of twelve plots organized in a four by three grid, and is summarized in Table 1. Each row of Figure 4 compares a sparse vs. a non sparse iRBM for the four types of regularization used. In general, the overall clustering performance of all models is positively affected by the introduction of a sparsity penalty. The average increase of the best AMI score of sparse models compared to non-sparse models is around 0.09, measured over all types of regularization as given in Table 1 . The number of hidden units is lower for sparse models compared to their non-sparse counterparts, depicted in the second column of Figure 4. The mean number of hidden units after half of the training iterations and at the end is given in Table 1 and shows that the sparse models only need a fourth of the amount of hidden units than the non-sparse models at 500 epochs while reaching higher scores. This difference grows even further when reaching the end of the training at 1000 epochs, where non-sparse models have around five times more hidden units than the sparse models have. The reason for this is that the sparsity penalty makes the model learn representations which use fewer hidden units. This means that the training sample's patterns are learned by fewer filters. Each of them has to be more expressive compared to a non-sparse model, where the amount of active hidden units is only limited by the size of the hidden layer. Due to Assumption (ii) the smaller size of hidden layers limits as well the number of identified clusters. The deployment of sparsity reduces the number of identified clusters by $58 \%$, on average taken over all types of regularization at their best ARI score, see the last column of Table 1. For the non-sparse models, the number of identified clusters increases continuously while training progresses (Figure 4, third column, blue lines) reaching an average of 98 units at 1000 epochs. Whereas the number of identified clusters of the sparse models is 24 on average at their best score. The increased number of found clusters does lower the clustering scores of the non sparse models, see (Figure 4, first column, blue lines). This behavior can as well be observed when comparing the mean scores of sparse and non sparse models at 500 and 1000 epochs, stated in Table 1.

However, the increase in performance of the sparse models can be explained by the fact that fewer hidden units relate to fewer learned filters. When the number of filters stays the same during training, they will learn a better representation of the data which translates to a better clustering, as indicated by higher AMI scores.

Figure 5 illustrates the influence of different types of regularization on an iRBM with sparsity penalty, namely $L_{0}, L_{1}, L_{2}$ and $L_{12}$, on the clustering performance (top), the size of the hidden layer (middle) and the number of identified clusters (bottom). The top plot of Figure 5 shows that $L_{1}$ and $L_{12}$ regularizations slightly enhance the clustering performance at the end of the first quarter of the considered training span. This behavior reflects the sparsity inducing properties of $L_{1}$ regularization. These are as well present in $L_{12}$ regularization, where they reduce the number of active parameters, and with that, decrease the number of training iterations (epochs) needed to optimize these fewer parameters. The plot also shows that this advantage vanishes at the end of the second quarter and the performance of all models stays almost unchanged for the last half of the training.

The second plot of Figure 5 (middle) shows the corresponding hidden layer sizes of the models assessed in the upper plot. As a first observation, the adaptive property of the iRBM is illustrated by the varying numbers of hidden units over the course of the training procedure. Additionally, it can be seen, that in the first half of epochs the $L_{2}$ regularized model has a smaller hidden layer size than models with other types of regularization. This reflects the $L_{2}$ specific smoothening influence on the weights. This property, which is arising from the limiting $\beta$ penalty of Equation (7), counteracts the for growing necessary filter specialization. The plot also shows a sudden increase in hidden units after approximately 500 epochs for $L_{1}$ and $L_{2}$ regularized models. The reason for this behavior is 

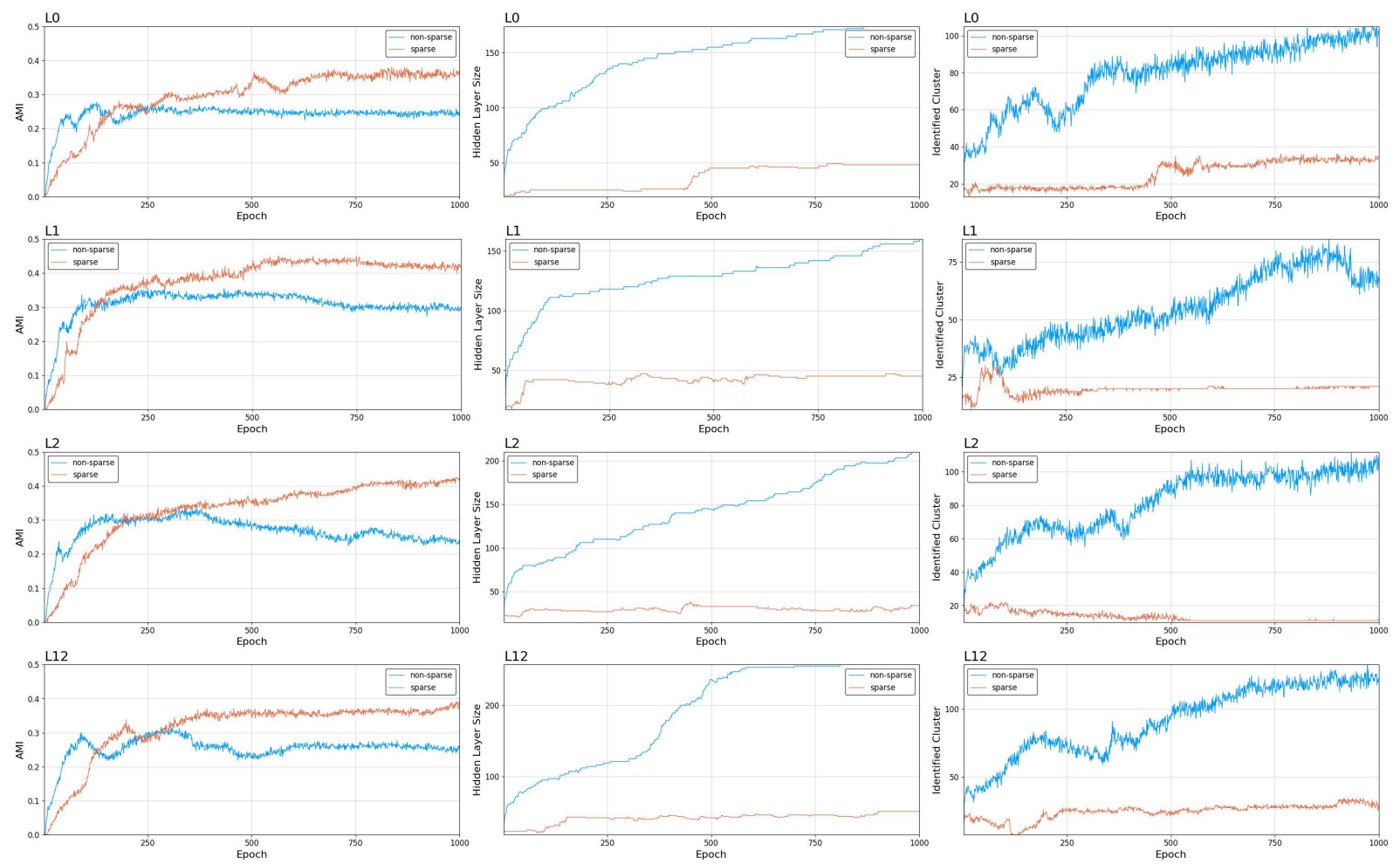

Figure 4: Grid of twelve plots having three plots in each of the four rows, where AMI, hidden layer size and number of identified clusters are depicted respectively for two models: one trained without sparsity penalty (blue lines) and a model trained with sparsity penalty (red lines). In each row, the two models depicted have been trained with one of the following types of regularization: $L_{0}$ (first row), $L_{1}$ (second row), $L_{2}$ (third row) and $L_{12}$ (fourth row).

Table 1: Mean values of AMI, hidden layer size and number of identified clusters of models trained with and without sparsity after 500 and 1000 epochs and at their best (maximal) ARI score.

\begin{tabular}{|c|c|c|c|c|c|c|c|c|c|}
\hline \multirow{2}{*}{ models } & \multicolumn{3}{|c|}{ at 500 epochs } & \multicolumn{3}{|c|}{ at 1000 epochs } & \multicolumn{3}{|c|}{ at best AMI scores } \\
\hline & $\begin{array}{c}\text { mean } \\
\text { AMI score }\end{array}$ & $\begin{array}{c}\text { number of } \\
\text { hidden units }\end{array}$ & $\begin{array}{c}\text { number of } \\
\text { found clusters }\end{array}$ & $\begin{array}{c}\text { mean } \\
\text { AMI score }\end{array}$ & $\begin{array}{l}\text { number of } \\
\text { hidden units }\end{array}$ & $\begin{array}{c}\text { number of } \\
\text { found clusters }\end{array}$ & $\begin{array}{l}\text { mean best } \\
\text { AMI score }\end{array}$ & $\begin{array}{c}\text { number of } \\
\text { hidden units }\end{array}$ & $\begin{array}{c}\text { number of } \\
\text { found clusters }\end{array}$ \\
\hline non-sparse & $0.27 \pm 0.02$ & $166 \pm 24$ & $82 \pm 11$ & $0.26 \pm 0.01$ & $200 \pm 22$ & $98 \pm 11$ & $0.32 \pm 0.02$ & $118 \pm 5$ & $57 \pm 6$ \\
\hline sparse & $0.36 \pm 0.02$ & $40 \pm 3$ & $22 \pm 4$ & $0.40 \pm 0.01$ & $45 \pm 4$ & $24 \pm 5$ & $0.41 \pm 0.02$ & $44 \pm 4$ & $24 \pm 5$ \\
\hline
\end{tabular}

that the training method finds a better parameter setting ${ }^{3}$ leading to more discriminative filters and allowing the model to grow further.

The third plot of Figure 5 (bottom) depicts the found number of clusters. As already encountered in the first and second experiments does the number of identified clusters vary in the initial training phase, but stabilizes when the AMI (top plot) converges towards its maximum value. As shown in Table 1 , the number of found clusters is very similar among the sparse models trained with different regularization settings except for $L_{2}$ throughout the training process (low standard deviation values). Most notably is the large increase

\footnotetext{
${ }^{3}$ This is indicated when looking at the reconstruction error of the considered models. It can be seen that the $L_{1}$ and $L_{2}$ models' error decreases further after platooning for approx. 250 epochs.
}

in the number of clusters of the $L_{2}$ regularized model while only a small increase is registered for the $L_{1}$ regularized model. The reason for this is twofold, firstly not all hidden units will automatically form a cluster, according to Assumption (ii) made in Section 4 , that implies that only dominant units will determine a cluster. Secondly, type $L_{1}$ regularization implies, as described in Section 2, sparsity which leads to fewer but larger weights allowing better discriminative filters than the weight smoothening properties of $L_{2}$ regularization. The large increase in clusters of the $L_{2}$ regularized model results as well in a slight drop in clustering performance (top, Figure 5). The experiment showes, that the sparse iRBM settles 

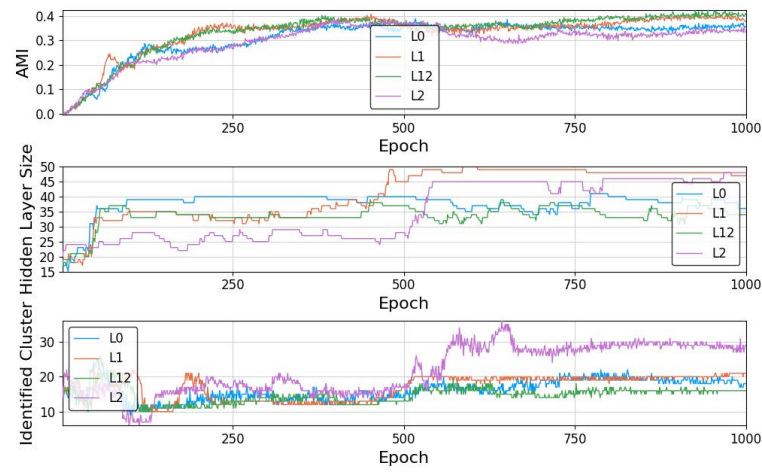

Figure 5: Each plot depicts a behavioral indicator of four models during training (1000 epochs) evaluated on a test set. Each of the four given models is trained using one of following types of regularization: $L_{0}$ (blue line), $L_{1}$ (orange line), $L_{2}$ (violet line) and $L_{12}$ (green line). (top) Clustering performance expressed using the AMI. (middle) Number of units in the hidden Layer. (bottom) Number of found clusters.

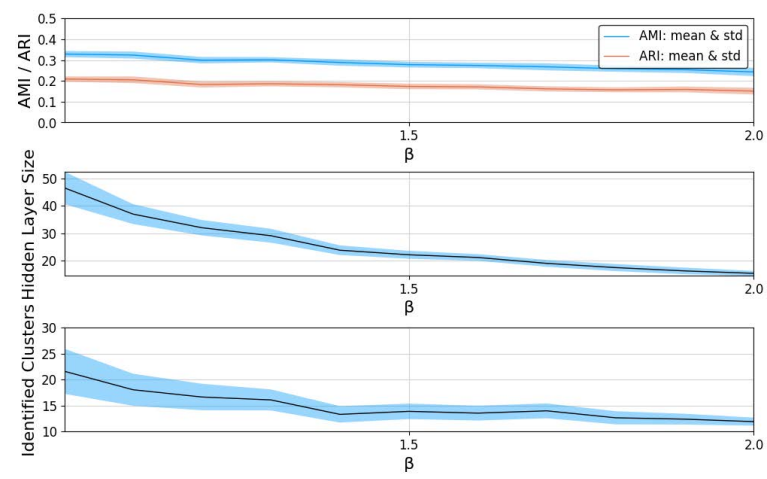

Figure 6: (top) The plot depicts clustering performance of iRBMs with different $\beta$ settings expressed using AMI (blue) and ARI (red). Mean (line) and standard deviation (shade) based on five evaluations per configuration. (mid) Mean (line) and standard deviation (shade) of the number of corresponding units in the hidden layer. (bottom) Mean (line) and standard deviation (shade) of the corresponding number of identified clusters. Results of 50 evaluations per setting. All models have been trained for 250 epochs on 2500 samples of the binarized MNIST dataset. A sparsity penalty but no regularization is applied. The $\beta$ parameter is varied.

with a hidden layer size within the recommended range of 25 to 50 units identified in the second experiment for the standard RBM.

Experiment 4. A core parameter of the $\mathrm{iRBM}$ is $\beta$ which enables the model's adaptive behavior. As Côte and Larochelle point out is the iRBM relatively insensitive to its choice and " $\beta$ 's role is only to ensure the iRBM is properly defined" [3]. Figure 6 illustrates the iRBM's clustering performance for a range of settings for $\beta$. As expected, a higher $\beta$ value reduces the number of hidden units as well as the number of clusters (Figure 6, mid and bot), since it increases the penalty term in Equation (9) and thus forces the model to learn better filters in order to grow. Better filters are more expressive and will be dominant for a broader range of samples and thus effectively decrease the total number of identified clusters ${ }^{4}$, see (Figure 6, bottom).

Interestingly, the confidence interval for the slope of a linear regression line (fitted on the AMI) is between -0.066 and -0.099 with a confidence level of $99.995 \%$. This means that, while the number of clusters decreases towards the true number of labels with increased $\beta$, the clustering performance is not increasing as one might expect. While the number of clusters approaches the true number of labels, the performance reduces, i.e., the model's misclassification rate increases. The too few hidden units limit the representational capabilities of the iRBM and lead to (extreme) cases where one or two clusters contain most of the test samples, which has a negative effect on the measured clustering performance. The results indicate that $\beta$ should be chosen close to one to achieve maximal clustering performance. On the other hand, setting $\beta$ below 1.1 leads to a high number of identified clusters, which might reduce the interpretability of the produced clustering, whereas $\beta$ settings above above 1.4 hinders the model's representational capabilities. Therefore we set $\beta=1.1$ for all experiments, since it provides a reasonable tradeoff between clustering performances and number of identified clusters.

\section{DISCUSSION}

The conducted experiments have shown, that the iRBM is able to find common patterns within the data as well as grouping them into a reasonable number of clusters. In this section, the produced clustering of the iRBM is discussed using example outputs, which showcase typical clustering results for the MNIST data set. Figure 7 depicts the clustering results of 1000 samples of an iRBM in the form of a confusion matrix. Each row corresponds to the true label of the samples and each column to the assigned cluster, which in this case is 25. The color of each cell indicates the amount of samples occurring at the specific configuration of labels and clusters; darker corresponds to more samples. Ideally, there would be one dark cell in each column which means that entities in this cluster are mostly from the same label.

However, in Figure 7 we see that the model does not reproduce the true labeling of the data. For example, the first cluster (first column) contains mostly zeros but also some eights. On the other hand clusters 2-3 contain almost exclusively ones. We see as well, that cluster 10, 11 and 12 are very small with less than 5 samples. The reason for this might be that the pattern that these clusters learned from the training samples is not present in the test samples.

Figure 8 shows five clusters in greater detail. As it can be seen, samples grouped into the same cluster (A, B, C, D or E) illustrated in Figure 8 share similarities with samples from respective clusters.

As illustrated in Figure 8(a) and (b), the model is able to find (in some cases) clusters that reflect very well the true labeling of the data: Cluster A contains only samples labeled as ones, whereas cluster B contains a mixture of true labels: $85 \%$ ones, $5 \%$ fours, $7.5 \%$ sevens and $2.5 \%$ nines. Both, cluster A and B have learned a very

${ }^{4}$ Additionally to the limitation of the number of hidden units arising with the deployment of Assumption (ii). 


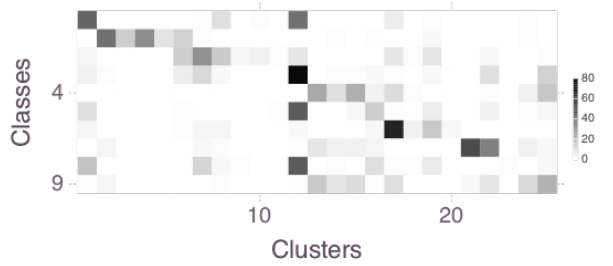

Figure 7: Confusion Matrix summarizing the clustering result of 1000 samples clustered by an iRBM with deployed sparsity trained for 1000 epochs on 2500 samples. Each cell $c_{i j}$ depicts the number samples of true class $i$ (row) and assigned cluster $j$ (column). A darker cell color corresponds to a higher sample count.

similar pattern which has a strong emphasis on a vertical stroke. The stroke is slightly leaned to the right for cluster A and slightly bend (or leaned) to the left for cluster B, which is as well reflected by the samples grouped in these clusters. This shows, on one side that the iRBM is able to differentiate between slightly altered versions of simple patterns, and on the other side, that multiple clusters may represent the same true label. The case of clusters A and B, where the true labels do not reflect different angles in which the number one can be written, explains the resulting higher number of clusters (24 on average) compared to the number of true labels (10).

The two clusters illustrated by Figure 8 (c) and (d) embody a more complex pattern than the one reflected in clusters $\mathrm{A}$ and $\mathrm{B}$. The filter associated with cluster $\mathrm{C}$ (Figure 8(c)) shows a pattern having a horizontal stroke and an angled vertical stroke. The true labels of samples grouped in cluster C are mostly (98\%) sevens ${ }^{5}$.

The iRBM does not always output as good clusters as depicted in Figure 8(d). If a pattern, shared among different numbers and therefore belonging to several true class labels, has been learned then the resulting cluster will consist of multiple true class labels. The cluster illustrated by Figure 8(d) consists, among others, out of $38 \%$ fours, $9 \%$ sevens and $50 \%$ nines. However, the test samples grouped in this clusters share similarities among each other, e.g., a vertical line with something attached to its upper left side.

The variety of true labels within the same cluster can be explained by the filter associated with the dominant unit for this cluster, shown in Figure 8 (bottom, right of each subplot). This behavior can be observed in cluster E, which corresponds to the thirteenth column of the confusion matrix given in Figure 7 . In this cluster zeros, threes, fives, and eights are grouped together due to the fact that they exhibit the common pattern of having a round stroke in the bottom right and top left.

Although the clustering is not always according to the assigned true labels, the samples contained in one cluster depict a common pattern which separates them from other samples, e.g., numbers with round strokes are separated from numbers with straight and leaned numbers. Assumption (ii) is the main reason for this behavior. The restriction to a single unit hinders the model to differentiate between minor changes of complex patterns, which might have been recognized by a composition of hidden units.

${ }^{5}$ The single test sample that is found in this cluster which is not labeled as a seven is the fist sample in the last row of Figure 8(c), which is labeled as six.

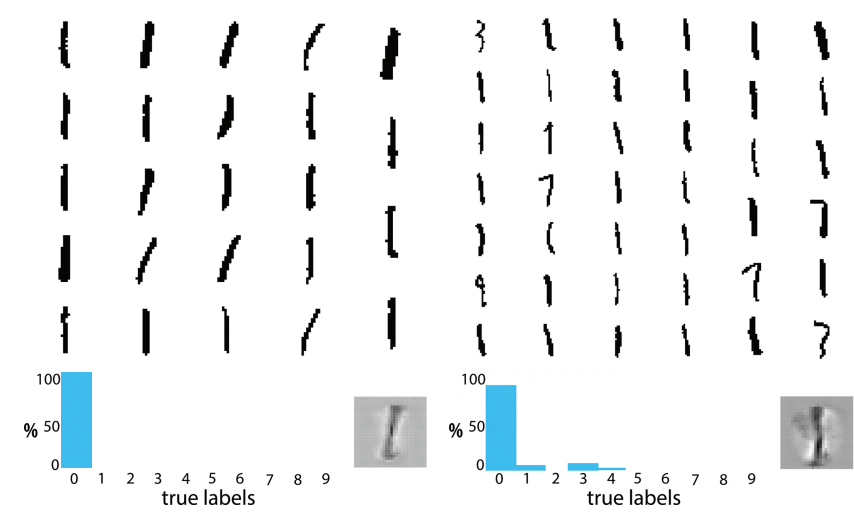

(a) Cluster A

(b) Cluster B

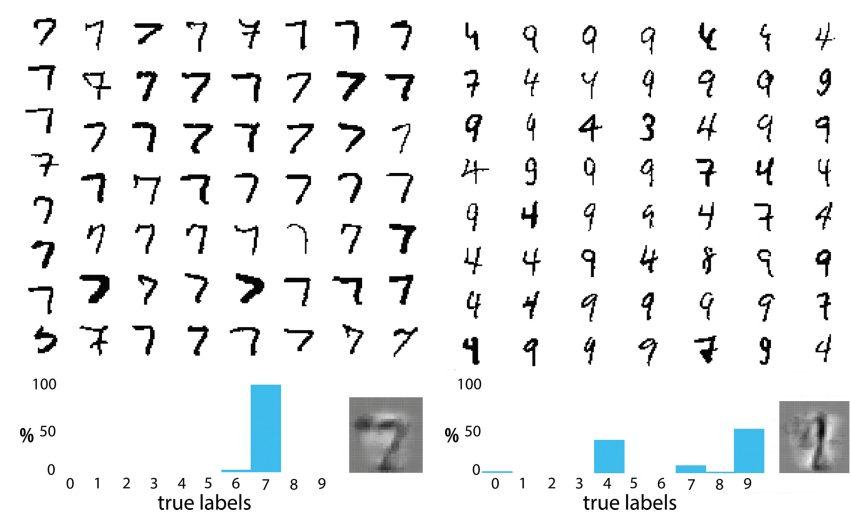

(c) Cluster C

(d) Cluster D

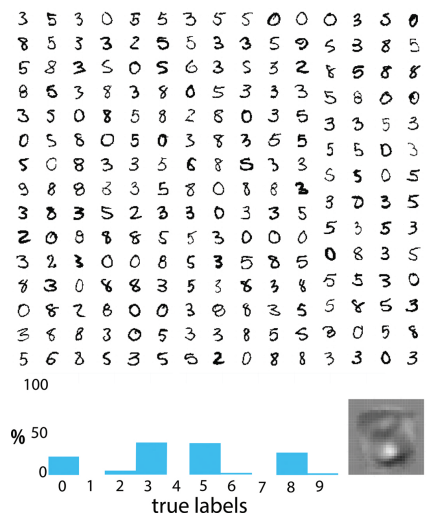

(e) Cluster E

Figure 8: Resulting clusters summarizing a single cluster in each subplot (a), (b), (c), (d), (e). Each subplot shows (top half) all test samples that were assigned to this cluster, (bottom left) a bar chart depicting the occurrence of the true labels within the cluster in percentage and (bottom right) the filter associated with the dominant unit of the given clusters. The test set contains 1000 randomly chosen samples which were not included in the training. 


\section{SUMMARY AND CONCLUSIONS}

In this paper, the iRBM has been deployed to the problem of clustering. To enable the use of the iRBM as a self-contained clustering method two assumptions, i.e., (i) samples embodying similar patters will be associated with similar hidden states and (ii) that it is sufficient to consider the most active (dominant) hidden unit to define a sample's cluster membership, have been introduced and discussed. The introduced assumptions allow the use of the iRBM as a clustering method by enabling the model to differentiate between patterns that have been learned in an unsupervised manner. Especially the choice of Assumption (ii) maintains the model's adaptive behavior and successfully extends the iRBM to a self-contained clustering approach, while at the same time limits the number of identified clusters.

Multiple experiments have been conducted exploiting the clustering behavior of the iRBM, granting insight into the interactions between different regularization and sparsity penalties under the introduced assumptions. The conducted experiments showed that deploying a sparsity penalty increased the iRBM's clustering performance. The sparsity penalty reduced the size of the hidden layer, and thereby the number of identified clusters while increasing the clustering performance. The study of the influence of the iRBM's $\beta$ parameter, which enables the model's self-regulating characteristics by allowing the model to adapt its hidden layer size, showed that higher values of $\beta$ lowered the AMI score significantly. Based on our experiments a $\beta$ value around 1.1 seems to be an appropriate choice. However, further studies involving other data have to be conducted to substantiate this claim. The iRBM with deployed sparsity penalty was able to learn on average around 24 strong (dominant) filters when deployed to the binarized MNIST dataset.

In our future research, we will study how one can utilize the iRBM for identifying more complex patterns as previously discussed in Section 6. Compositions of hidden units or deep structures as used by Salama et al. [26] are interesting approaches to represent/learn more complex patterns. However, the chosen approach must not cancel out the self-adjusting characteristics of the iRBM. Comparing the iRBM's clustering performance against baseline approaches such as DPMM could as well be part of future studies.

\section{REFERENCES}

[1] Charles E. Antoniak. 1974. Mixtures of Dirichlet Processes with Applications to Bayesian Nonparametric Problems. The Annals of Statistics 2, 6 (1974), 1152-1174.

[2] Christopher M Bishop. 2006. Pattern Recognition and Machine Learning. Vol. 4 738 pages.

[3] Marc-Alexandre Côté and Hugo Larochelle. 2016. An Infinite Restricted Boltzmann Machine. Neural computation (2016).

[4] Asja Fischer. 2015. Training Restricted Boltzmann Machines. KI - Künstliche Intelligenz August 2014 (2015), 2-5.

[5] B. J. Frey and D. Dueck. 2008. Clustering by Passing Messages Between Data Points. Science 315, 5814 (2008), 972-976.

[6] Chen Fuqiang, Wu Yan, Bu Yude, and Zhao Guodong. 2014. Spectral Classification Using Restricted Boltzmann Machine. Publications of the Astronomical Society of Australia 31 (2014), e001.

[7] Peter V Gehler, Pgehler Tuebingen M P G De, Alex D Holub, Holub Vision, Caltech Edu, Max Welling, Welling Ics, and U C I Edu. 2006. The Rate Adapting Poisson Model for Information Retrieval and Object Recognition. (2006), 337-344.

[8] G Goos, J Hartmanis, and J Van Leeuwen. 1999. When Is Nearest Neighbor Meaningful? (1999).

[9] A D Gordon. 1999. Classification. x, 256 p. pages.

[10] Trevor Hastie, Robert Tibshirani, and Jerome Friedman. 2009. The Elements of Statistical Learning. Elements 1 (2009), 337-387.
[11] Geoffrey Hinton. 2002. Training Products of Experts by Minimizing Contrastive Divergence. 1800 (2002), 1771-1800.

[12] Geoffrey Hinton. 2006. A Fast Learning Algorithm for Deep Belief Nets. 1554 (2006), 1527-1554.

[13] Geoffrey Hinton and Ruslan Salakhutdinov. 2006. Reducing the Dimensionality of Data with Neural Networks. Science 313, 504 (2006).

[14] Lawrence Hubert and Phipps Arabie. 1985. Comparing partitions. Fournal of Classification 2, 1 (1985), 193-218.

[15] S. B. Kotsiantis, I. D. Zaharakis, and P. E. Pintelas. 2006. Machine learning: A review of classification and combining techniques. Artificial Intelligence Review 26, 3 (2006), 159-190.

[16] Hugo Larochelle and Yoshua Bengio. 2008. Classification using discriminative restricted Boltzmann machines. Proceedings of the 25th International Conference on Machine Learning (ICML 08) (2008), 536-543.

[17] Hugo Larochelle, Michael Mandel, Razvan Pascanu, and Yoshua Bengio. 2012. Learning Algorithms for the Classification Restricted Boltzmann Machine. The Journal of Machine Learning Research 13 (2012), 643-669.

[18] Yann LeCun, Yoshua Bengio, and Geoffrey Hinton. 2015. Deep learning. Nature 521, 7553 (2015), 436-444.

[19] Yann LeCun, Léon Bottou, Yoshua Bengio, and Patrick Haffner. 1998. Gradientbased learning applied to document recognition. Proc. IEEE 86, 11 (1998), 22782323.

[20] Shixia Liu, Xiting Wang, Yangqiu Song, and Baining Guo. 2015. Evolutionary bayesian rose trees. IEEE Transactions on Knowledge and Data Engineering 27, 6 (2015), 1533-1546.

[21] Vinod Nair and Ge Hinton. 2009. 3D Object Recognition with Deep Belief Nets. Advances in Neural Information Processing Systems (2009), 1-9.

[22] Chapelle Olivier, Bernhard Schölkopf, and Alexander Zien. 2006. Semi-Supervised Learning. Vol. 1. 524 pages.

[23] Marc Aurelio Ranzato, Y-Lan Boureau, and Yann Lecun. 2008. Sparse Feature Learning for Deep Belief Networks. Advances in neural information processing systems (NIPS) (2008), 1185-1192.

[24] Peter J. Rousseeuw. 1987. Silhouettes: A graphical aid to the interpretation and validation of cluster analysis. F. Comput. Appl. Math. 20, C (1987), 53-65.

[25] Ruslan Salakhutdinov, Andriy Mnih, and Geoffrey Hinton. 2007. Restricted Boltzmann Machines for Collaborative Filtering. (2007).

[26] Mostafa Salama. 2010. Deep belief network for clustering and classification of a continuous data. In Signal Processing and Information Technology (ISSPIT), 2010 IEEE International Symposium on. Vol. 3. IEEE, 473--477.

[27] Jorge M. Santos and Mark Embrechts. 2009. On the use of the adjusted rand index as a metric for evaluating supervised classification. Lecture Notes in Computer Science (including subseries Lecture Notes in Artificial Intelligence and Lecture Notes in Bioinformatics) 5769 LNCS, PART 2 (2009), 175-184.

[28] Tanya Schmah, Geoffrey Hinton, Richard S Zemel, Steven L Small, and Strother S C. 2009. Generative versus discriminative training of RBMs for classification of fMRI images. Advances in Neural Information Processing Systems 21 (2009), 1409-1416.

[29] Paul Smolensky. 1986. Information processing in dynamical systems: Foundations of harmony theory. Parallel Distributed Processing Explorations in the Microstructure of Cognition 1, 1 (1986), 194-281.

[30] Kihyuk Sohn, Dae Yon Jung, Honglak Lee, and Alfred O. Hero III. 2011. Efficient Learning of Sparse, Distributed, Convolutional Feature Representations for Object Recognition. IEEE International Conference on Computer Vision (2011), 2643-2650.

[31] H Joe Steinhauer, Alexander Karlsson, and Tove Helldin. 2016. Root-Cause Localization using Restricted Boltzmann Machines. (2016).

[32] Thomas S Ferguson. 1973. A Bayesian Analysis of Some non-parametric problems. The Annals of Statistics 1, 2 (1973), 209-230.

[33] R Tibshirani, G Walther, and T Hastie. 2001. Estimating the number of clusters in a data set via the gap statistic. (2001), 411-423 pages.

[34] Nguyen Xuan Vinh, Julien Epps, and James Bailey. 2010. Information theoretic measures for clusterings comparison: Variants, properties, normalization and correction for chance. The fournal of Machine Learning Research 11 (2010), 28372854 .

[35] Junyuan Xie, Ross Girshick, and Ali Farhadi. 2016. Unsupervised Deep Embedding for Clustering Analysis. In Proceedings of The 33rd International Conference on Machine Learning. 478-487.

[36] Rui Xu. 2005. Survey of Clustering Algorithms. IEEE Transactions on Neural Networks 16, 3 (2005), 645-678.

[37] T Xu, Zhongfei Mark Zhang, Philip S Yu, and B Long. 2008. Dirichlet Process Based Evolutionary Clustering. 2008 Eighth IEEE International Conference on Data Mining - ICDM'08 (2008), 648-657.

[38] Shenghuo Zhu, Tao Li, and M. Ogihara. 2002. An Algorithm for Non-Distance Based Clustering in High Dimensional Spaces. TR763 (2002), 52.

[39] Hui Zou and Trevor Hastie. 2005. Regularization and variable selection via the elastic net. Journal of the Royal Statistical Society. Series B: Statistical Methodology 67, 2 (2005), 301-320. 